\title{
CHEQUEO CONSTRUCTIVO DE LAS FACHADAS DE MADRID/ESPAÑA
}

\author{
(CHEKING OF BUILDING FAÇADES IN MADRID/SPAIN)
}

Juan Monjo Carrió, Dr. Arquitecto

\section{RESUMEN}

El presente articulo describe el estudio estadistico sobre patología constructiva de fachadas urbanas que está llevando a cabo el Departamento de Construcciones Arquitectónicas de la Universidad Politécnica de Madrid, con sede en su Escuela Técnica Superior de Arquitectura.

Se indican tanto los objetivos del estudio, como el método propuesto, del que se han dado ya algunos de los pasos, y se hace especial hincapie en las fichas de toma de datos, base del trabajo, asi como en la clasificación de lesiones, materiales, elementos constructivos, etc.

Por último, se presentan los primeros resultados estadísticos obtenidos, mediante una ficha de prueba, durante el curso 1986/87.

\section{SUMMARY}

The article describes the estatistic study on construction pathology of urban facades that is being done by the Department of Construction of the Politechnic University of Madrid, at the School of Architecture.

The objectives of the study are indicated, as well as the proposed method, part of wich has already been developed, and special emphasize is done on the data counters, base of the work, as well as in the classification of damages, construction materials, constructive elements, etc.

Finally, the first statistic results, obtained through a proof counter during 1987, are offered.

\section{INTRODUCCION: Antecedentes y estado actual del tema.}

La patología constructiva como el estudio de las lesiones que aparecen en los edificios, sus sintomas, sus causas y sus remedios, es un tema actual en el mundo de la Edificación; y lo es, no sólo por la conflictividad que aporta, tanto para los usuarios como para los técnicos que intervienen en el proceso constructivo en cualquiera de sus fases (diseño, fabricación de materiales, construcción y dirección de obra) sino, además, por la orientación actual hacia la rehabilitación y uso de edificios antiguos como una consecuencia de la nueva legislación urbanistica, por un lado, y del aumento del nivel cultural de los ciudadanos, por otro, que comprende la importancia de la conservación del patrimonio arquitectónico.
Dos factores marcan, pues, la necesidad de los estudios de Patología Constructiva. Por una parte, la propia problemática de la integridad de los edificios y la permanencia de sus características mecánicas, funcionales y estéticas a lo largo de su vida, lo que condiciona su mantenimiento y, por otra, la nueva problemática de la rehabilitación, que conlleva la recuperación del edificio y sus partes hasta actualizar su funcionamiento constructivo según su nuevo uso.

Esta preocupación se inició, a nivel internacional, en la década de los 60 y, como siempre, se empezó por el estudio patológico de los elementos estructurales, por su incidencia en la propia seguridad del edificio afectado. Por ello, la mayoria de publicaciones en esa década hablan de estructuras. En la década de los 70 la preocupación se ha hecho extensiva al resto de los 
elementos constructivos del edificio, sobre todo fachadas, cubiertas y acabados, que es el tema que nos afecta. Existen ya numerosos estudios sobre esta rama de la Patología Constructiva, aunque prácticamente todos ellos se reducen a un estudio de diversos casos según una secuencia típica que pasa por las fases de analizar los sintomas, definir la lesión y plantear el remedio. Sin embargo, son escasos los estudios estadísticos reales sobre los tipos de lesiones más frecuentes, o los tipos de causas y sus relaciones, si exceptuamos el trabajo llevado a cabo en 1973/74 por el Bureau Securitas y Secotec, en Francia, en el que analizan 2.979 lesiones de todo tipo en aquel país, asi como sus causas, y un estudio llevado a cabo por el Departamento de Valladolid, dirigido por el autor de este artículo y publicado por esa universidad en mayo de 1987 en un libro titulado "Patologia en fachadas urbanas" y en el que antes de entrar en el estudio de casos concretos, se realiza un breve análisis estadístico de la problemática de la Patología Constructiva de la ciudad de Valladolid. Asimismo, hay que mencionar el artículo publicado en esta misma revista, en su n. ${ }^{0} 364$ de octubre de 1984, titulado "Patología de la Construcción en España: aproximación estadístican, en el cual se resume el estudio de 586 informes sobre obras con lesiones en estructura o cerramientos, que sirve de base para un análisis estadístico en el que se refleja la incidencia de las distintas causas posibles en la patología de las estructuras de nuestro pais.

Por otra parte, la mayoría de las publicaciones en español sobre este tema son traducciones de otras publicaciones extranjeras, aunque el citado interés creciente se ha concretado ya en nuestro país en un $1^{\text {er }}$ Congreso Nacional de Patología de la Construcción, organizado por el Colegio Oficial de Arquitectos de $\mathrm{Ca}$ taluña, en marzo de 1985, en el transcurso del cual ya indiqué la necesidad y el interés de un trabajo como el que ahora se presenta, con un amplio estudio estadístico representativo del estado patológico en nuestras fachadas urbanas, con una cuantificación de los tipos de lesiones más normales y sus causas para que sirva de base, tanto a los técnicos del sector en su trabajo cotidiano, como a los estudiosos del problema, en número satisfactoriamente creciente día a día, sobre todo en el nivel universitario e investigador en el que me muevo.

\section{OBJETIVOS}

Como consecuencia de esta situación y de las novedades que se preven en el futuro próximo, el Departamento de Construcciones Arquitectónicas de la Universidad Politécnica de Madrid se ha propuesto, y ha iniciado, el trabajo que aqui se expone, para lo que se han fijado los siguientes objetivos:
- Conocer estadísticamente las lesiones constructivas más corrientes (cuantificadas) que aparecen en las fachadas urbanas de Madrid, ordenadas por zonas.

- Conocer las causas más comunes (cuantificadas) de dichas lesiones en relación con la zona; la edad del edificio, sus caracteristicas constructivas y sus condiciones ambientales.

- Conocer la influencia, en la aparición de las lesiones, de los siguientes factores, por porcentajes y correlaciones estadísticas:

- Edad del edificio y su uso.

- Estado de conservación de la fachada.

- Técnica constructiva (sistema, material, etc.).

- Contaminación ambiental.

- Orientación geográfica de la fachada.

- Diseño de la misma.

- Nivel de exposición y protección al viento.

- Conocer y analizar las reparaciones efectuadas, su frecuencia y su resultado.

En definitiva, tener un planteamiento general de la problemática de la patología constructiva de las fachadas urbanas de una gran ciudad, en una determinada situación geográfica, con datos estadísticamente válidos para que el colectivo profesional del sector de la construcción de edificios (proyectistas, fabricantes de materiales, constructores, directores de obra, promotores y usuarios) tengan un conocimiento claro del problema y una base de datos cierta que les sirva en su actuación profesional, tanto para mejorar sus proyectos (evitando caer en los mismos errores, mejorando el diseño) como sus obras (mejorando las calidades de los materiales y la ejecución de las obras, previendo un adecuado mantenimiento, etc.) como para enfocar correctamente la rehabilitación de edificios antiguos y, en definitiva, mejorar la imagen de la ciudad.

Asimismo, este estudio puede servir como pauta para otros similares en otras ciudades, ya que con ello se ofrece al técnico un conjunto de datos necesarios para su labor al igual que se hace en otros campos, como la medicina, la alimentación, etc. y ser usada como base de datos para todos aquellos estudiosos de la construcción de fachadas urbanas, su patología y su restauración.

\section{METODO}

Para llevar a cabo el trabajo se plantean una serie de etapas, la primera de las cuales ya se ha iniciado. Podemos distinguir tres etapas básicas, cada una de ellas dividida, a su vez, en fases: 


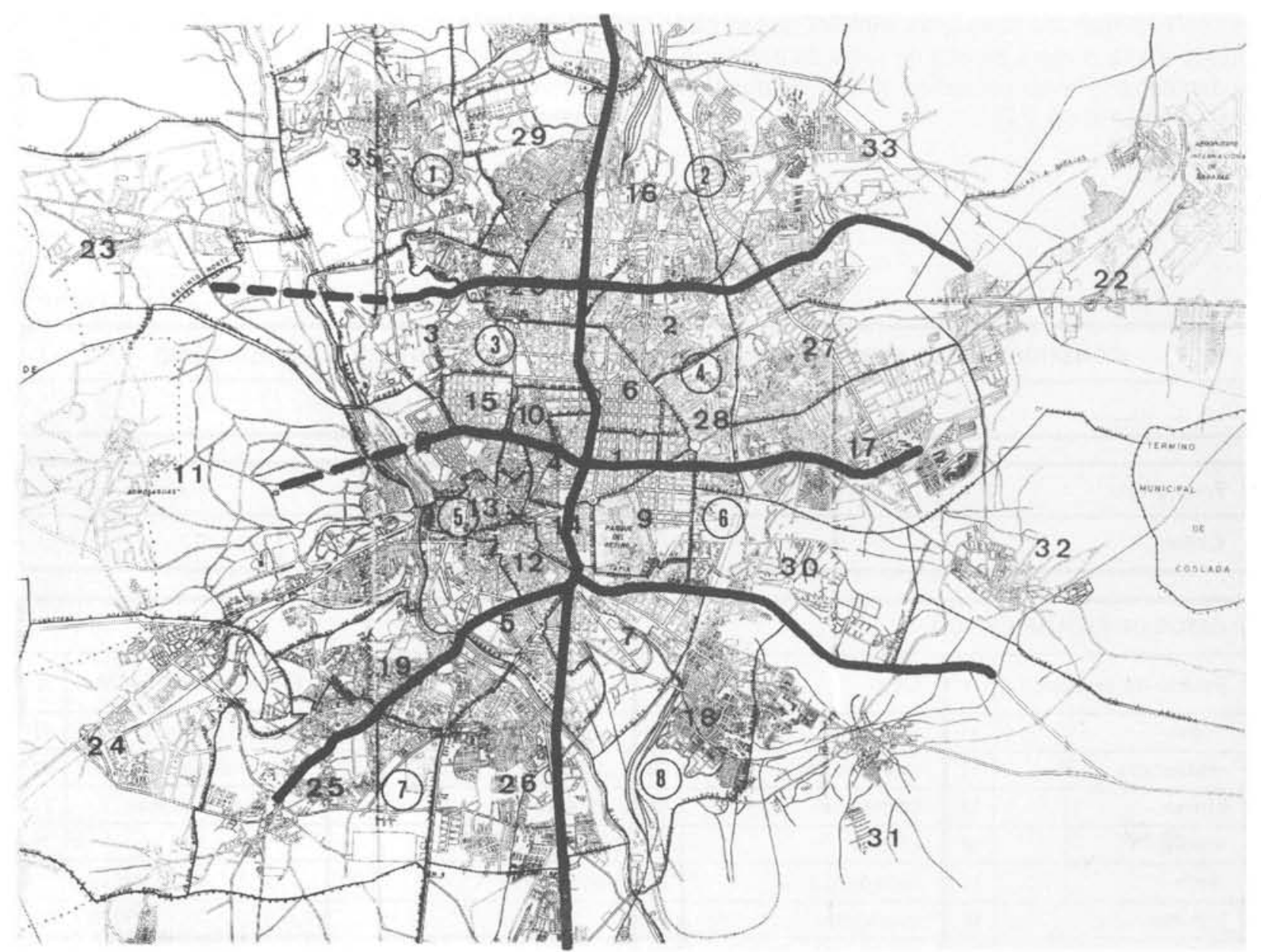

Fig. 1

- Toma de datos.

- Ensayos de laboratorio.

- Análisis estadísticos y conclusiones.

\subsection{Toma de datos}

Es la etapa fundamental del trabajo, pues de ella depende la fiabilidad y la representatividad de los resultados finales.

\subsubsection{Preparación de observadores}

Para asegurar la fiabilidad hay que disponer de un equipo de observadores técnicamente preparados y, a la vez, objetivos. Se ha confiado esta responsabilidad a alumnos de 6. ${ }^{\circ}$ Curso de la Escuela T.S. de Arquitectura de Madrid, convenientemente dirigidos y coordinados por los profesores de prácticas de la asignatura de Construcción IV de dicha Escuela, que figuran como investigadores del equipo. Los alumnos han recibido lecciones teóricas a lo largo del curso sobre $\mathrm{Pa}$ tología y, en cualquier caso, se han dedicado las primeras semanas a prepararlos adecuadamente con ejemplos prácticos, clasificaciones teóricas y algunas visitas de observación.

\subsubsection{Descomposición zonal}

Se ha descompuesto la ciudad en ocho zonas, según se indica en el plano de la Fig. 1, para lo que se han tenido en cuenta, entre otras, las siguientes condiciones:

- Zonificación por contaminación ambiental según datos del Excmo. Ayuntamiento de Madrid.

- Zonificación por situación geográfica.

- Zonificación por distribución económico-social.

Se prevén un mínimo de 8 zonas. Esta fase puede ser simultánea a la anterior.

\subsubsection{Confección de fichas}

Resulta una fase realmente importante en todo el proceso, ya que la ficha es una herramienta fundamental para la obtención correcta y uniforme de los datos, dado el elevado número de observadores necesario.

Hasta el momento, y como se comenta más adelante, se han preparado las fichas (2) correspondientes a la primera toma de datos, para lo cual ha sido necesa- 
rio confeccionar una ficha teórica inicial, que se ha sometido a una primera prueba de toma de datos y que ha demostrado la necesidad de utilizar las dos fichas que se adjuntan ( 1 y 2 ).
Más adelante se atacará la confección de la (o las) fichas correspondientes a la segunda toma de datos, asi como las sucesivas que resultan necesarias en el desarrollo del trabajo.

FICHA

CONSTRUCCIONES ARQUITECTONICAS IV - ESCUELA T. S. ARQUITECTURA - U. P. MADRID

CHEQUEO CONSTRUCTIVO DE FACHADAS DE MADRID

\begin{tabular}{|l|l|l|}
\hline Zona-grupo: & Fecha: & \multicolumn{2}{|c|}{$/$} \\
\hline Calle: & & N.o: \\
\hline
\end{tabular}

\begin{tabular}{|c|c|c|c|c|c|c|c|c|c|c|c|c|c|c|}
\hline \multicolumn{15}{|c|}{ DATOS DE IDENTIFICACION } \\
\hline \multicolumn{4}{|c|}{ Estado de conserv. } & 1 & \multicolumn{3}{|c|}{ Uso } & 2 & \multicolumn{3}{|l|}{ Edad } & \multicolumn{2}{|c|}{ Existencia de lesión } & 4 \\
\hline \multicolumn{4}{|c|}{ nueva } & 11 & \multicolumn{3}{|c|}{ res. prop. hor. } & 21 & \multicolumn{2}{|l|}{ ant. 1800} & 31 & \multicolumn{2}{|c|}{ sin lesiones } & 41 \\
\hline \multicolumn{4}{|c|}{ restaurada } & 12 & \multicolumn{3}{|c|}{ res. alquiler } & 22 & \multicolumn{2}{|l|}{$1800-1900$} & 32 & \multicolumn{2}{|c|}{ con una lesión } & 42 \\
\hline \multicolumn{4}{|c|}{ buena } & 13 & \multicolumn{3}{|c|}{ comercial } & 23 & \multicolumn{2}{|l|}{$1900-1940$} & 33 & \multicolumn{2}{|c|}{2 ó más lesiones } & 43 \\
\hline \multicolumn{4}{|c|}{ media } & 14 & \multicolumn{3}{|c|}{ oficina } & 24 & \multicolumn{2}{|l|}{$1940-1970$} & 34 & & & \\
\hline \multicolumn{4}{|c|}{ mala } & 15 & \multicolumn{3}{|c|}{ enseñanza } & 25 & \multicolumn{2}{|l|}{ post. 1970} & 35 & & & \\
\hline \multicolumn{4}{|c|}{ pésima } & 16 & \multicolumn{3}{|c|}{ industria } & 26 & & & & & & \\
\hline & & & & & \multicolumn{3}{|c|}{ mixto } & 27 & & & & & & \\
\hline \multicolumn{5}{|c|}{ Nivel de contaminación } & 5 & \multicolumn{3}{|c|}{ Orientación } & & 6 & \multicolumn{3}{|c|}{ Materiales principales } & 7 \\
\hline \multicolumn{2}{|l|}{$\mathrm{SO}_{2}$} & \multicolumn{2}{|l|}{ Part. } & \multicolumn{2}{|l|}{$\mathrm{CO}$} & $\mathrm{N}$ & 61 & \multicolumn{2}{|l|}{$\mathrm{S}$} & 65 & ladr. v. & 71 & piedra mac. & 76 \\
\hline alto & 51 & alto & 54 & alto & 57 & NE & 62 & so & & 66 & enf. + pint. & 72 & piedra cha. & 77 \\
\hline med. & 52 & med. & 55 & med. & 58 & $E$ & 63 & 0 & & 67 & revoco & 73 & horm. in si & 78 \\
\hline bajo & 53 & bajo & 56 & bajo & 59 & SE & 64 & NO & & 68 & pla. cer. & 74 & hor. pref. & 79 \\
\hline & & & & & & & & & & & metal & 75 & madera & 710 \\
\hline
\end{tabular}

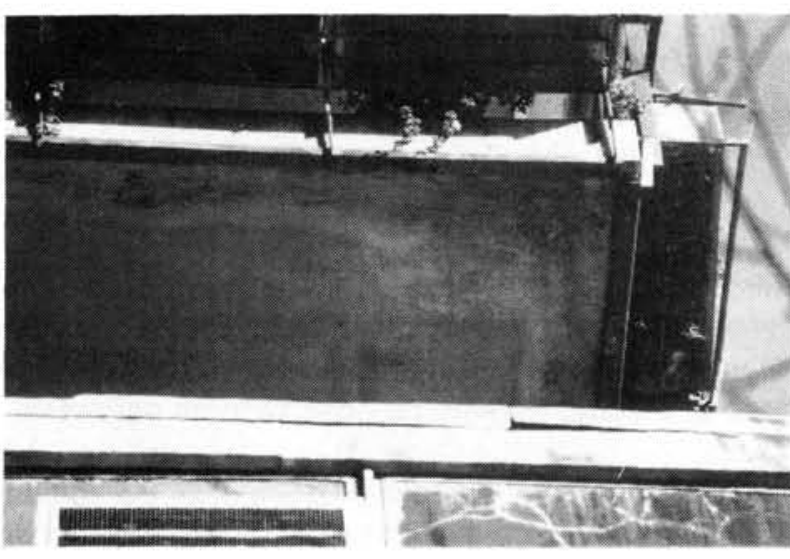

Humedad en enfoscado y pintura del intradós del balcón.

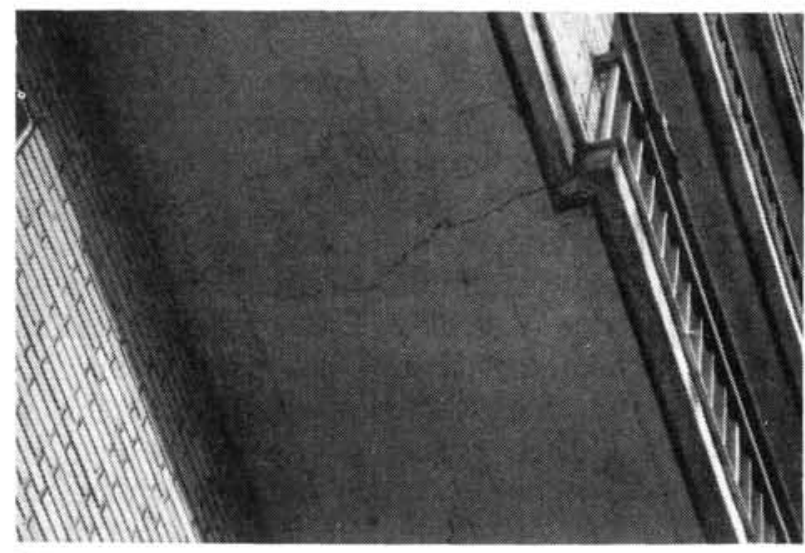

Grieta en hormigón in situ en el intradós del balcón. 


\begin{tabular}{|c|c|c|c|}
\hline \multicolumn{4}{|c|}{ CONSTRUCCIONES ARQUITECTONICAS IV - ESCUELA T.S. ARQUITECTURA - U. P. MADRID } \\
\hline \multicolumn{4}{|c|}{ CHEQUEO CONSTRUCTIVO DE FACHADAS DE MADRID } \\
\hline Zona-grupo: & $/$ & Fecha: & / \\
\hline Calle: & & & N.: \\
\hline
\end{tabular}

\begin{tabular}{|c|c|c|c|c|c|c|c|c|c|c|c|}
\hline \multicolumn{8}{|c|}{ DATOS DE LA LESION } & \multicolumn{4}{|l|}{ FICHA N. } \\
\hline \multicolumn{3}{|l|}{ Lesión } & \multirow{2}{*}{$\frac{10}{106}$} & \multicolumn{3}{|l|}{ Material } & \multirow{2}{*}{$\begin{array}{l}20 \\
208\end{array}$} & \multicolumn{3}{|c|}{ Elemento constructivo } & \multirow{2}{*}{$\frac{30}{307}$} \\
\hline humedad & 101 & eflor. & & ladr. v. & 201 & pied. m. & & zócalo & 301 & mold. v. & \\
\hline grieta & 102 & corro. & 107 & enfoscado & 202 & pied. ch. & 209 & paño ciego & 302 & mold. $\mathrm{h}$. & 308 \\
\hline fisura & 103 & sucied. & 108 & revoco & 203 & pla. ce. & 210 & cornisa & 303 & estr. vis. & 309 \\
\hline desprend. & 104 & organ. & 109 & estuco & 204 & pintu. & 211 & barandilla & 304 & ele. suel. & 310 \\
\hline \multirow[t]{3}{*}{ erosión } & 105 & & & h. in situ & 205 & metal & 212 & balcón & 305 & general & 311 \\
\hline & & & & h. prefa. & 206 & madera & 213 & ventana & 306 & & \\
\hline & & & & plástico & 207 & & & & & & \\
\hline \multicolumn{3}{|c|}{ Localización } & 40 & \multicolumn{3}{|c|}{ Nivel de exposición } & 50 & & & & \\
\hline $0.4 \mathrm{~m}$ & 401 & central & 405 & \multicolumn{3}{|l|}{ Ex-1a } & 501 & & & & \\
\hline $4-10 \mathrm{~m}$ & 402 & lateral & 406 & \multicolumn{3}{|l|}{$E x-1 b$} & 502 & & & & \\
\hline $10 \mathrm{~m}$ & 403 & esqu. & 407 & \multicolumn{3}{|l|}{$E x-2 a$} & 503 & & & & \\
\hline gene. v. & 404 & gen. $h$. & 408 & \multicolumn{3}{|l|}{$E x-2 b$} & 504 & & & & \\
\hline
\end{tabular}

\subsubsection{Primera toma de datos}

Se organizan grupos de 2 ó 3 observadores (para asegurar la objetividad de la observación) que recorren las distintas zonas tomando nota de todas las lesiones observables y rellenando las correspondientes fichas. En esta primera fase se toman datos de:

- Identificación, recogiendo el estado de conservación de la fachada, uso del edificio, edad, existencia de lesiones, nivel de contaminación de la zona, orientación de la fachada y sus materiales de construcción principales, además de las fotos necesarias.

- Lesión, rellenando una ficha por cada lesión distinta y recogiendo los datos correspondientes a materiales y elementos constructivos afectados, localización de la lesión dentro de la fachada y nivel de exposición de la misma. Con el fin de homogeneizar en lo posible la toma de datos, se provee a los observadores de una "guia", para cumplimentar am. bas fichas, en la que se explica cada uno de los apartados y cuyo texto se adjunta en el anejo n. 1.

\section{ANEJO 1}

CONSTRUCCION IV - CURSO $87 / 88$ CHEQUE CONSTRUCTIVO DE FACHADAS DE MADRID

\section{TOMA DE DATOS}

\section{Guía para cumplimentar la ficha de DATOS DE IDENTIFICACION}

Se rellenará una ficha por cada una de las fachadas que componen la calle asignada, recorriéndola por manzanas en ambos lados.

1. Estado de conservación. Se refiere a la fachada, no al edificio en conjunto.

11. nueva, con menos de 3 años de existencia.

12. restaurada, dentro de los últimos 3 años.

13. buena, con más de 3 años y sin lesiones.

14. media, con más de 3 años y sin lesiones graves.

15. mala, con más de 3 años, con alguna lesión grave y sin conservación.

16. pésima, con más de 3 años y en muy mal es. tado. 
2. Uso. Alguno de los indicados. Si el mismo edificio combina más de uno, mixto.

3. Edad. Alguna de las indicadas.

4. Existencia de lesión. Indicar una de las 3 opciones.

5. Nivel de contaminación. De la zona. Se indicará el nivel de cada una de las tres columnas $\left(\mathrm{SO}_{2}\right.$, Par. tículas, $\mathrm{CO}$ ) de acuerdo con los mapas en poder de la cátedra.

6. Orientación. Del plano de la fachada donde aparece la lesión. Si es distinto del conjunto de la fachada, indicarlo.

7. Materiales principales. Indicar los más representativos de la fachada, hasta un máximo de 3.

\section{Guia para cumplimentar la ficha de DATOS DE LA LESION}

Se rellenará una ficha por cada tipo de lesión dentro de una fachada, aunque haya varias lesiones diferentes. Incluso, si para un mismo tipo de lesión en una fachada hay casos suficientemente distintos, se rellenará una ficha por cada uno.

10. Lesión. Se refiere al síntoma, no a su posible causa.

101. humedad, manchas de humedad de infiltración, tanto vivas como muertas.

102. grietas, las que afectan a todo el elemento constructivo.

103. fisuras, las que afectan a la superficie del mismo.

104. desprendimiento, tanto de elementos sueltos (plaquetas, baldosas, etc.) como de elementos continuos (revocos, pinturas, etc.).

105. erosión, debida a impactos, roces o acción climática.

106. eflorescencia, incluso la formación de sales por acción de la contaminación.

107. corrosión, sobre metales y sus efectos sobre el soporte.

108. suciedad, producida por la acción combinada de contaminación, viento y lluvia, en forma de churretones, pátina general, etc.

109. organismos, tanto animales (monos, insectos) como vegetales (hongos, líquenes, etc.).

20. Material. Se refiere al material de la fachada al que afecta la lesión, pudiendo reflejar hasta 3.

201. ladrillo visto, fábricas de este material.

202. enfoscado, de mortero de cemento o cemento y cal.
203. revoco, acabado de mortero de cemento con tratamiento especial.

204. estuco, acabado de mortero de yeso (antiguo) normalmente decorativo.

205. hormigón in situ

206. hormigón prefabricado, tanto en grandes paneles como en pequeñas piezas de "piedra artificial".

207. plástico, elementos (normalmente sueltos) de ese material.

208. piedra maciza, fábricas de piedra.

209. piedra chapada, aplacados de piedra.

210. plaqueta cerámica.

211. pinturas, independientemente del soporte sobre el que están aplicadas.

212. metal, tanto de carpinterias, como de cerrajeria, como de elementos sueltos.

213. madera, tanto en carpintería, como de cerrajería, como de elementos sueltos.

30. Elemento constructivo. Se refiere al elemento componente de la fachada sobre el que aparece la lesión, hasta un máximo de 3.

301. zócalo, cuando existe con material diferente del resto. En cualquier caso, inferior a $3 \mathrm{~m}$ de altura.

302. paño ciego, en cualquier punto de la fachada.

303. cornisa, remate superior de la fachada.

304. barandilla, peto vertical de terraza o balcón, sea macizo o de elementos lineales.

305. balcón o mirador, cualquier cuerpo volado de la fachada, sobre todo la parte horizontal inferior.

306. ventana, carpintería y su hueco (dintel, jambas y alféizar).

307. moldura vertical, sobresaliente o rehundida en la fachada.

308. moldura horizontal, sobresaliente o rehundida en la fachada.

309. estructura vista, tanto horizontal como vertical.

310. elementos sueltos, decorativos o funcionales (bajantes, canalones).

311. general, cuando afecta a más de 3 elementos.

40. Localización. Se refiere a la situación más destacada de la lesión dentro de la fachada. Se deberá indicar, tanto en sentido vertical (1. a columna, 401 al 404 ) como en sentido horizontal ( 2 . $^{\text {a columna, }} 405$ al 408). 
Esta primera toma de datos se va a llevar a cabo en dos o tres años sucesivos, dada la necesidad de su acople con los cursos académicos. Así, en este primer curso $87 / 88$ se está realizando la primera etapa, ya prácticamente finalizada, en la que se han rellenado las $\mathrm{fi}$ chas de identificación correspondientes a unas 2.000 fachadas distribuidas entre las 8 zonas mencionadas, y unas 3.000 fichas de lesiones aparecidas en estas fachadas. Para ello se han lanzado a la calle unos 5 grupos de dos observadores por cada zona.

En el curso pasado (86/87) se realizó una toma de unas 400 lesiones con objeto de probar el funcionamiento de la primera ficha de toma de datos que se confeccionó. Estas lesiones nos permitieron alcanzar dos objetivos importantes: por un lado, llegar a la confección de las 2 fichas que se están utilizando actualmente. Por otro, realizar una primera estadística orientati- va que se comenta en el siguiente apartado.

\subsubsection{Primer análisis estadístico}

Como quiera que el objetivo final del trabajo es el conocimiento estadístico de la situación patológica de las fachadas de Madrid, para to que es fundamental el disponer de un amplio y correcto "banco de datos", un paso fundamental es confeccionar un buen programa analítico de base de datos que nos permita archivar fácilmente los datos que se van obteniendo y poderlos recuperar posteriormente agrupados en las distintas formas que nos sugieran los tipos de estadistica que necesitemos en cada caso. Para ello se hace necesaria esta fase del primer análisis estadístico, que nos permitirá la correcta confección del programa analíti$\mathrm{co}$ e, incluso, las correcciones convenientes en las fichas de toma de datos.

FICHA DE PRUEBA

DEPARTAMENTO DE CONSTRUCCIONES ARQUITECTONICAS IV

ETSA.UPM

\begin{tabular}{|l|l|} 
CHEQUEO CONSTRUCTIVO DE FACHADAS DE MADRID & Ficha N.
\end{tabular}

Primera toma de datos

Ficha N.

\begin{tabular}{|l|l|l|l|l|l|l|}
\hline Alumno & \multicolumn{3}{|c|}{} & Grupo & \\
\hline Situación & zona & & calle & & & \\
\hline Contaminación & nivel & & & tipo & & \\
\hline
\end{tabular}

\begin{tabular}{|c|c|c|c|c|}
\hline \multicolumn{4}{|c|}{ DATOS CONSTRUCTIVOS } & Fecha \\
\hline \multicolumn{2}{|r|}{ Material } & \multicolumn{2}{|c|}{ Elem. construc. } & Localización \\
\hline humedad & ladrillo & \multicolumn{2}{|c|}{ zócalo } & $0-3 \mathrm{~m}$ \\
\hline grietalfisura & enfoscado & \multicolumn{2}{|c|}{ paño ciego } & $3-9 m$ \\
\hline desprendimiento & revoco/estuco & \multicolumn{2}{|c|}{ cornisa } & $9 \mathrm{~m}$ \\
\hline erosión & horm. in situ & \multicolumn{2}{|c|}{ barandilla } & central \\
\hline eflorescencia & horm. prefab. & \multicolumn{2}{|c|}{ ventana } & lateral \\
\hline corrosión & piedra (maciz.) & \multicolumn{2}{|c|}{ baicón } & esquina \\
\hline suciedad & piedra (chapa) & \multicolumn{2}{|c|}{ moldu. vert. } & \\
\hline \multirow{4}{*}{ organismos } & plaquet. cera. & \multicolumn{2}{|c|}{ moldu. horiz. } & \\
\hline & metal & \multicolumn{2}{|c|}{ estruct. vista } & \\
\hline & madera & \multicolumn{2}{|c|}{ elem. sueltos } & \\
\hline & pinturas & & & \\
\hline \multicolumn{4}{|c|}{ DATOS AMBIENTALES } & Fecha \\
\hline Conservación & Nivel exposición & \multicolumn{2}{|c|}{ Orientación } & Edad edificio \\
\hline Nueva & Ex-1 & $\mathrm{N}$ & $\mathrm{s}$ & Ant. 18 \\
\hline Rehabilitada & Ex-2 & $N-E$ & S.O & $1800-19$ \\
\hline Media & Ex-3 & $E$ & 0 & $1900-19$ \\
\hline Mala & Ex-4 & $S-E$ & $\mathrm{~N}-\mathrm{O}$ & $1940-19$ \\
\hline Pésima & & & & 1970-ac \\
\hline
\end{tabular}




\section{ANEJO 2 - DATOS ESTADISTICOS}

\section{TOTALES DE LESIONES}

Humedad

Grietas/fisuras

Desprendimiento

Erosiones

Eflorescencias

Corrosiones

Suciedades

Organismos

Total

TOTALES DE ELEMENTOS CONSTRUCTIVOS

Zócalo

Paño ciego

20

Cornisa

Barandilla

Ventana

Balcón

Moldura vertical

Moldura horizontal

Estructura vista

Elementos sueltos

General

Total

TOTALES DE DATOS AMBIENTALES CONSERVACION

Nueva

Rehabilitada

17

Media

Mala

Pésima

Total

99

41

314

\section{ORIENTACION}

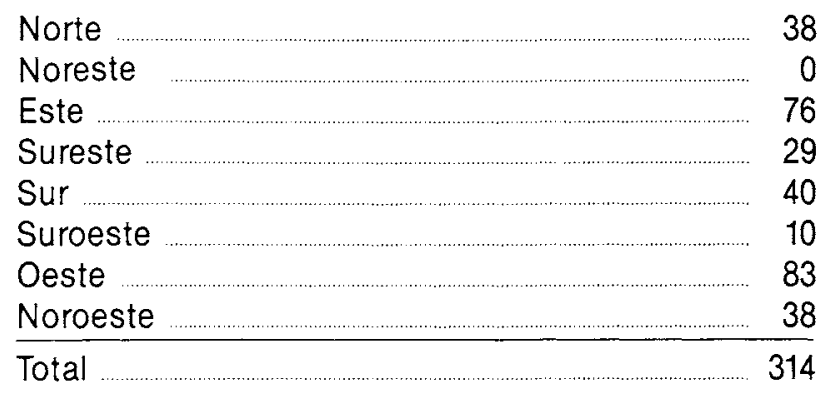
(se permitia marcar hasta 3 materiales por ficha)

TOTALES DE MATERIALES

(se permitia marcar hasta 3 materiales por ficha)

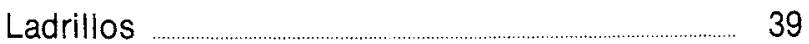

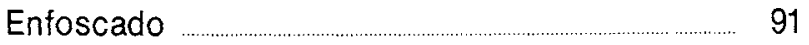

Revestimientolestuco ................................. 136

Hormigón in situ ............................. 2

Hormigón prefabricado _................................... 5

Piedra maciza $\quad 11$

Piedra chapada _....................... 31

Plaqueta cerámica ............................ 7

Metal _............................................

Madera $\ldots$

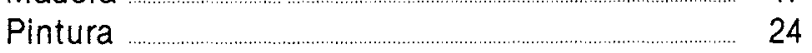

Total 380

\section{TOTALES DE LOCALIZACIONES}

\section{Vertical}

De 0 a $3 \mathrm{~m} \quad 46$

De 3 a $9 \mathrm{~m} \ldots \ldots \ldots \ldots . .102$

Más de $9 \mathrm{~m} \ldots \ldots$

General _...... 58

Horizontal

Central $\quad 117$

Lateral $\quad 66$

Esquina $\quad 33$

General ..._._.

Total $\ldots \ldots \ldots \ldots$

\section{NIVEL DE EXPOSICION}

Nivel 1

Nivel 2

Nivel $3 \quad 0$

Nivel $4 \ldots \ldots \ldots \ldots$

Total

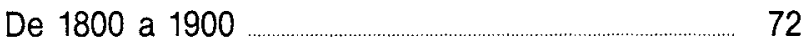

De 1900 a $1940 \ldots \ldots \ldots$

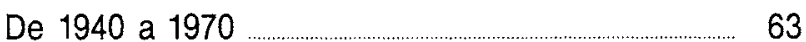

De 1970 a $1987 \ldots \ldots$

Total …_. 314 
TOTAL DE HUMEDADES POR MATERIALES

Ladrillo

Enfoscado

Revoco/estuco

Piedra chapa

Madera

Total

TOTAL DE DESPRENDIMIENTOS POR MATERIALES

Ladrillo
Enfoscado

39

$\begin{array}{ll}\text { Revocolestuco } & 67\end{array}$

Piedra maciza $\quad 2$

$\begin{array}{ll}\text { Piedra chapa } & 7\end{array}$

Plaqueta cerámica $\quad 7$

Metal _. 4

Madera 6

\begin{tabular}{ll} 
Pintura $\quad 20$ \\
\hline$\longrightarrow \longrightarrow$
\end{tabular}

\section{DESPRENDIMIENTOS EN ENFOSCADOS}

EN PAÑO CIEGO

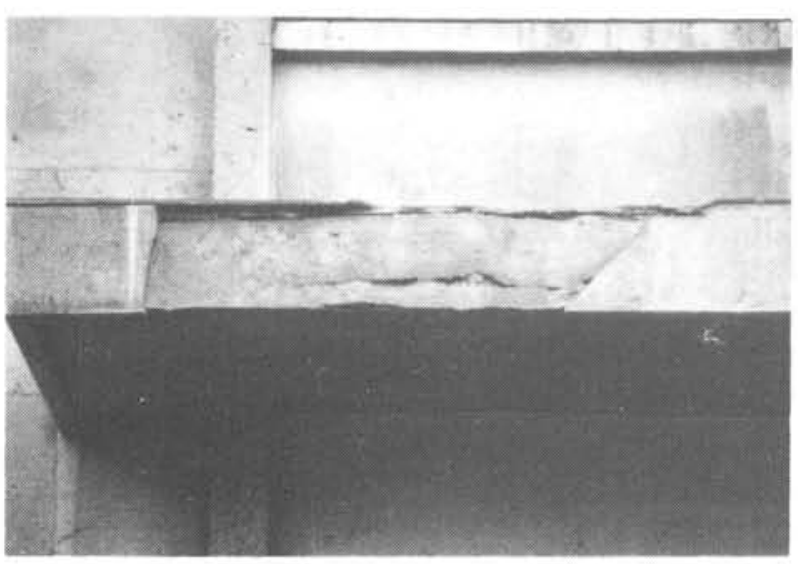

Desprendimiento del chapado de piedra en el borde del balcón.

El primer análisis se llevó a cabo en el pasado curso (86/87) con los datos de lesiones obtenidos con la mencionada "ficha de prueban. Se utilizó para ello una base de datos elemental ("open access") que nos permitió llegar a una primera estadística de los distintos datos contenidos en la ficha, así como combinaciones de ellos (ejemplo, en el anejo n. ${ }^{\circ} 2$ ).

Actualmente, con las conclusiones alcanzadas des. pués de aquel primer análisis, y ya con los nuevos datos de la primera etapa mencionados en el apartado anterior, se está confeccionando un nuevo programa de banco de datos utilizando la base de datos «D base 3 plus" que nos posibilitarán una estadistica más amplia y flexible. Ello nos permitirá, por un lado, disponer de ese banco de datos útil, tanto para técnicos profesionales, como para estudiosos del tema y, por otro, co. nocer los condicionantes y lesiones más representativos para estudios en la segunda toma de datos.

\subsubsection{Segunda toma de datos}

A la vista del resultado de la fase anterior, se llevará a cabo un estudio pormenorizado de las lesiones se.

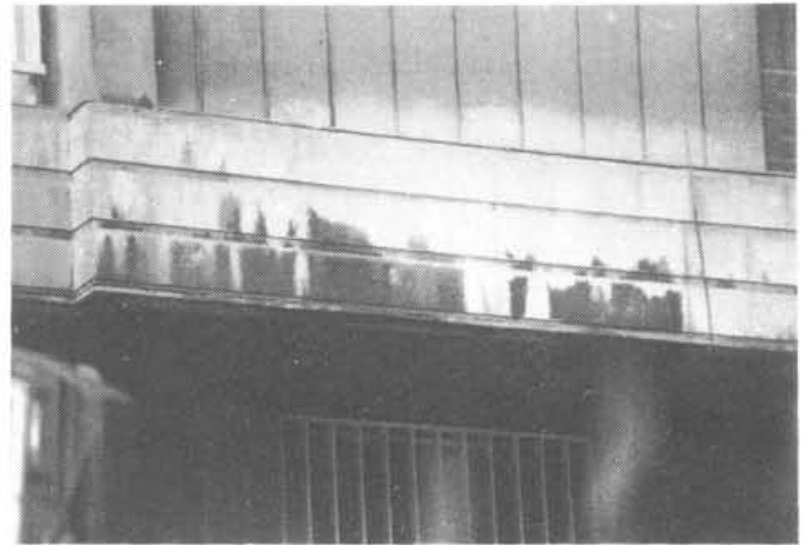

Suciedad en el chapado de piedra de un paño ciego.

leccionadas en las que, además de tomar muestras de los materiales afectados (dento de lo posible), se anotarán, por lo menos, los datos relativos a:

- Evolución general de la lesión.

- Diseño constructivo.

- Proceso constructivo.

- Reparaciones efectuadas o previstas.

- Mantenimiento del elemento constructivo afectado.

Se puede prever un número de casos a estudiar de 1.000 (10\% del total observado), de los cuales, en unos 500 de ellos, se podrán obtener muestras de materiales.

Con ello se procurará, además, llegar a conocer las causas directas e indirectas de la lesión, lo que nos permitirá, a su vez, alcanzar una estadistica complementaria de causas, similar a la alcanzada en otros trabajos e igualmente útil para técnicos y estudiosos. Para ello, se deberán dar los siguientes pasos:

- Confeccionar una ficha útil para esta segunda to. ma de datos, en la que se recojan los distintos ti. pos de causas y condicionantes posibles. 
- Preparar el banco de datos del ordenador para po. der archivar todos los que se recojan en esta fase y poderlos recuperar después según las necesida. des estadisticas.

Esta toma de datos se llevará a cabo, asimismo, en dos o tres cursos académicos sucesivos, paralelamente a la primera.
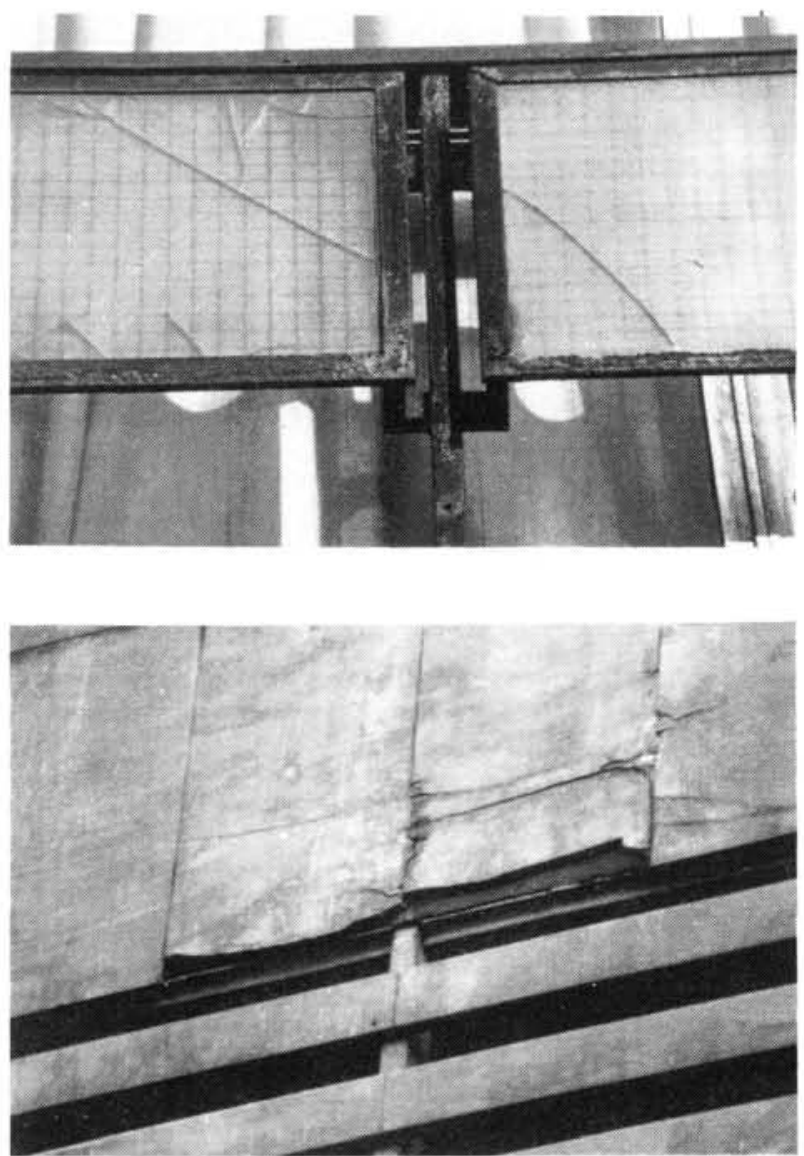

Desprendimiento de la piedra del chapado general.

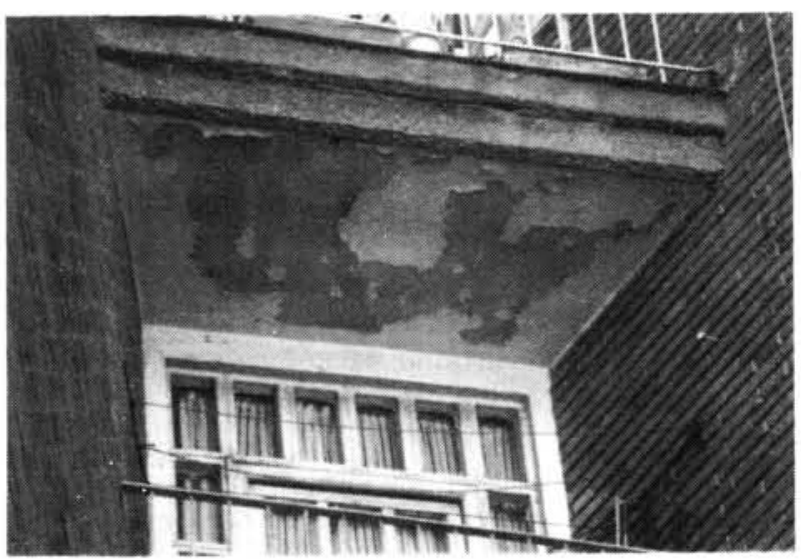

Desprendimiento de pintura en balcón.

Erosión en ladrillo visto (paño ciego).

\subsection{Ensayos de laboratorio}

Las muestras obtenidas en la segunda toma de datos se pretende que sean analizadas mediante los en. sayos físicos y químicos necesarios para saber su com. posición y características, lo que nos permitirá conocer, con mayor base científica, la evolución de las le. siones y sus origenes, con objeto de completar los datos de la fase anterior.

Aunque esta fase dependerá de las disponibilidades económicas del Departamento, se pretende que su realización se lleve a cabo en el propio laboratorio de materiales de la ETSAM, o bien en los que se ajusten a las necesidades de los ensayos en aquellos casos en que dicho laboratorio no disponga de la infraestructura necesaria para el análisis en concreto. En cualquier caso, es una fase necesaria si se quiere tener una certeza científica en muchos casos de lesiones, sobre to- 
do en aquellos en que tiene un papel importante algún tipo de mortero de acabado superficial, o en aquellas otras en las que se produzca una reacción química de alguno de los componentes de la fachada (eflorescencias, erosiones, etc.).

En definitiva esta fase es complementaria de la segunda toma de datos y, concretamente, de su correspondiente análisis estadístico.

\subsection{Análisis estadistico y conclusiones}

Con los datos tomados y los resultados de los ensayos se procederá al análisis estadístico definitivo que nos permitirá alcanzar las conclusiones finales del trabajo. Para ello, y de acuerdo con los objetivos planteados, se tratarán de obtener, por lo menos, los siguientes datos:

- Lesiones más comunes en las fachadas de Madrid, expresados en porcentajes.

- Materiales y elementos constructivos más deteriorados, expresados en porcentajes.

- Correlaciones entre ambos.

- Agrupación de ambos tipos de datos en función de factores ambientales e históricos, expresados en índices de correlación de los porcentajes de lesiones y materiales con los de dichos factores (orientación, nivel de exposición, etc.).

Todos estos datos se expresarán en forma analítica y gráfica, obteniéndose curvas representativas de todos ellos.

En cualquier caso nos quedará un banco de datos, permanentemente actualizable, que permitirá otros tipos de estudios estadísticos que resulten necesarios a estudiosos y técnicos.

\section{ESTADO ACTUAL DEL TRABAJO}

Tal como se ha comentado en los párrafos del apartado anterior, hasta el momento se han dado los si. guientes pasos:

- Confección de una primera ficha de toma de datos con la que se han recogido los correspondientes a unas 400 lesiones y que ha servido para el diseño de las fichas definitivas.

- Confección de las dos fichas para la primera toma de datos (datos de identificación y datos de lesión).
- Primera etapa de la primera toma de datos (en ejecución).

- División de la ciudad de Madrid en zonas (8 zonas).

- Zonificación de Madrid según los datos de contaminación ambiental facilitados por el Excelentísimo Ayuntamiento de Madrid.

- Preparación de un programa de archivo informático de prueba para el análisis estadístico de los primeros datos de lesiones.

- Confección del programa definitivo de archivo informático para la creación del banco de datos de patología de fachadas urbanas de Madrid (en proceso de confección).

A partir de ahora, una vez terminados la toma de datos y el programa de archivo, se procederá a constituir el banco de datos y a realizar nuevas tomas de datos en cursos académicos sucesivos. Paralelamente se realizará un primer análisis estadístico de estos datos.

Asimismo se procederá a la segunda toma de datos para realizar el análisis de causas, para lo cual se confeccionará la correspondiente ficha o fichas. Esta toma de datos, que se realizará también en sucesivos años académicos, irá acompañada de la toma de muestras de materiales y los correspondientes ensayos de laboratorio.

Una vez en posesión de todos estos datos se pasará a su análisis estadístico global y a la obtención de las conclusiones pertinentes, conclusiones que confiamos nos permitan alcanzar los objetivos que nos hemos propuesto y que figuran en el punto 2 . de este artículo. Concretamente esperamos alcanzar, por lo menos, los siguientes datos, según la fase:

- Del análisisis estadístico del banco de datos patológicos:

- Porcentajes de aparición de las distintas lesio. nes estudiadas.

- Porcentajes de incidencia de la patología en los diversos materiales de fachada.

- Porcentajes de incidencia en los diversos elementos constructivos que componen la fachada.

- Localizaciones más comunes de las lesiones en las fachadas, tanto en vertical como en horizontal. 
- Incidencia del nivel de contaminación en la patologia de las fachadas.

- Distribución de cada uno de los tipos de lesiones estudiados entre los diversos materiales y elementos constructivos, asi como entre combinaciones de éstos.

- Porcentajes de edificios con lesiones (una o más) entre los estudiados.

- Porcentaje e incidencia del uso y la edad del edificio en la existencia de lesiones en los mismos.

- Incidencia del nivel de contaminación y de la orientación en la patología de las fachadas urbanas.

- Correlaciones entre el estado de conservación del edificio, su uso y edad, y la existencia de lesiones en el mismo.

- Correlaciones entre el estado de conservación con los niveles de contaminación y la orientación.

- De los ensayos de laboratorio y del segundo análisis estadístico:

- Porcentajes de las causas origen de las lesio. nes, tanto de las directas (lesiones previas, es. fuerzos mecánicos, agentes atmosféricos, etc.) como de las indirectas (proyecto, ejecución, material, mantenimiento).

- Porcentajes de restauraciones practicadas y su resultado.

- Porcentajes de necesidades de restauración y tipos de las mismas.

\section{publicaciones del IETcc / CSIC}

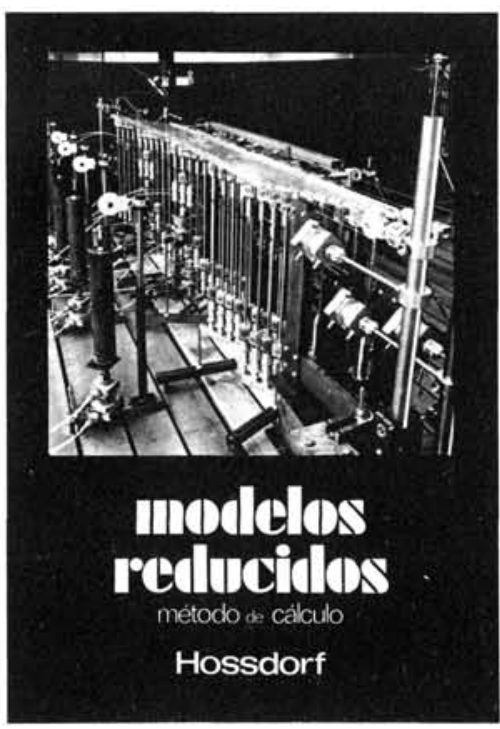

Modelos reducidos. Método de cálculo

H. Hossdorf, Ingeniero Civil

La técnica de los ensayos en modelos reducidos de estructuras sufre hoy dia una decisiva metamorfosis. estructuras surre hoy da una dio más bien de antesa Hasta hace pocompre era tomado en serio por los en serio por los acadérnicos teorizantes Dara comprender el comporta que se acudió las más de las veces. como que se acudio ultimo remedio debido a sus is veces, como a $r$ insulien. cias. Sin embaras a su conexion con los ordenadois digities, se ha trans. formado en un instrumento cienta en la váctica diaria del Ingeniero Proyectista. Un volumen encuadernado en cartoné plastificado
con lomo de tela, de $17 \times 24 \mathrm{~cm}$, compuesto de 250 páginas, 158 figuras y fotografias.

Precios: 1800 ptas : \$ USA 26.00

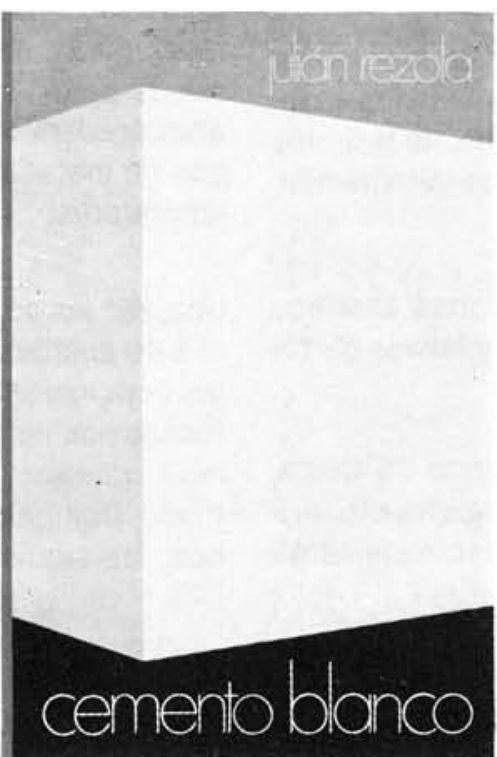

\section{Cemento blanco}

Julián Rezola

Ingeniero Quimico Dipl. I. Q. S.

Sabido es que existe una extensa y documentada bibliografia sobre el cemento gris: en cambio, no planco, ya que los escritos existentes se refieren solo a algunas peculiaridades que le distinguen de aquél.

El autor nos ofrece sus profundos conocimientos y su larga experiencia tanto en laboratorio como en fabricación.

La parte descriptiva del libro se complementa con gráficos, diagramas y fotografias de gran utilidad, destinados a conseguir la aplicación apropiada de este aglomerante

Un volumen encuadernado en cartoné policerado, de $17.4 \times 24.3 \mathrm{~cm}$, compuesto de 395 páginas, numerosas fiquras, tablas $y$ abacos

Precios: España, 1.700 ptas,; extranjero, \$ 24

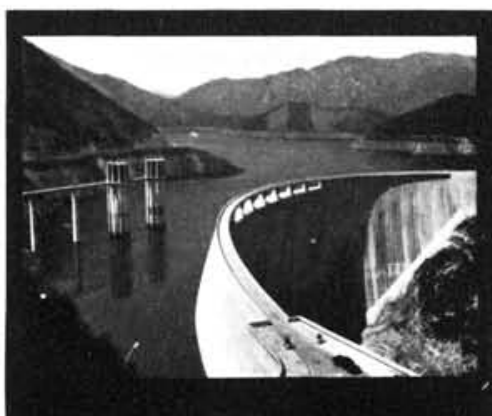

la presa bóveda de Susqueda

su proyecto construcción y comportamiento

A. Rebollo

La presa bóveda de Susqueda

A. Rebollo,

Dr. Ingeniero de Caminos

El esfuerzo del constructor de presas se sitủa. por su pretensión de perennidad, a contracorriente de las tendencias de la civilizacion actual, caracte rizada por lo fungible. Pueden evocarse las 10.000 grandes presas en funcionamiento o en construcción que están envejeciendo y reclaman los cuidados gerontológicos para mantener y perfeccionar su servicio y garantizar su inalienable pretensión de perennidad. En la medida en que todas nuevas obras, grandes o pequeñas, son portadoras de riesgos ecológicos y, a veces, catastroficos, que aumentan con el envejecimiento, la getontologia de las presas es todo un emplazo. La accion adelantada de Arturo Rebollo en este terreno marca un camino a seguir para todos los que aman su propia obra con la devoción paternal que el ha puesto en Susqueda.

Un volumen encuadernado en cartone plastificado con lomo de tela, de $18=24,5 \mathrm{~cm}$, compuesto de 408 páginas, 330 figuras y fotografias y 39 tablas Precios: 1.700 ptas : extranjero, \$ USA 2400 